\title{
A Physician's Guide to Working as a Locum Tenens
}

\author{
Mary M. Maniscalco, MD
}

Background: The number of physicians working as a locum tenens is increasing. Although most physicians who provide locum tenens coverage are often older and semiretired, an increasing number of physicians are taking this route for limited periods early in their careers.

Methods: The medical literature was searched through MEDLINE using the key words "career choice," "contract services," and "locum tenens." Information about locum tenens was gained by the author through research and a personal experience working as a locum tenens.

Results and Conclusion: Working as a locum tenens can be an opportunity to meet interesting patients, learn about local culture, see how practices are organized, learn adaptability, and broaden clinical skills. Opportunities are available through for-profit agencies, academic health centers, state agencies, federal sites such as the Indian Health Service, individual hospitals, and physicians or international staffing companies. Because the physician working as a locum tenens is an independent contractor, exercising some caution when choosing where to work by carefully checking workload and available resources is advised. (J Am Board Fam Pract 2003;16:242-5.)

A locum tenens, as defined by the American Heritage Dictionary, is a substitute physician or "one holding the place." Although practiced by physicians for decades in the United States and abroad, locum tenens did not become organized as an industry in the United States until the late 1970s or early 1980s. The commercial locum tenens industry grew from a demonstration project conducted at the University of Utah in the late 1970s. ${ }^{1}$ In 1981 an analysis of data from an 18-month study by the Health Systems Research Institute showed that it was cost-effective for rural physicians to hire a locum tenens to cover their practice while they were absent. ${ }^{2}$ Today, the number of physicians working as a locum tenens is growing, and sources currently estimate that $4 \%$ to $15 \%$ of physicians, at some point in their careers, will work in a locum tenens position. ${ }^{3,4}$

The reasons physicians work as a locum tenens are varied. Working as a short-term staff member can give physicians the opportunity to find out whether they want to work in a particular practice or new part of the country. Physicians who do not

Submitted, revised, 1 October 2002.

From the Department of Family Medicine (MMM), University of North Carolina at Chapel Hill. Address reprint requests to Mary M. Maniscalco, MD, UNC Department of Family Medicine, CB 7595 - Manning Dr, Chapel Hill, NC 27599. wish to work full-time, including those approaching retirement, might decide to take locum tenens positions. Others use this position during periods of transition, such as before starting a fellowship or after leaving a practice.

Although most physicians who provide locum tenens coverage are older and semiretired, an increasing number of physicians take this route for limited periods early in their careers. ${ }^{5}$ Primary care physicians still make up the largest number of locum tenens, but the number of other specialists is increasing because there are physician shortages in some specialty areas. ${ }^{6}$ Although locum tenens is usually a short-term career experience or option, some physicians do choose to become a permanent locum tenens, usually working for a commercial company.

The literature on locum tenens is limited, as are specific data related to the size and scope of the industry. The locum tenens industry is largely a private and highly competitive business in which information is not readily shared. It is difficult to learn about the quality of different locum tenens agencies, and there are limited data on the standards of care provided by locum tenens in general, although there is nothing to suggest the care is inferior. The purpose of this article is to provide an overview on the use of locum tenens and to serve as a practical resource for physicians evaluating potential locum tenens opportunities. 
Table 1. Resources for Locum Tenens Positions.

American Academy of Family Physicians:

www.aafp.org/practice/locumtenens.html

American College of Physicians: www.acponline.org

New England Fournal of Medicine Career Links: www.nejm.org/ careerlinks

National Association of Physician Recruiters and National Association of Locum Tenens Organization: www.napr.org or 800-726-5613

\section{Methods}

The medical literature was searched through MEDLINE using the key words "career choice," "contract services," and "locum tenens." Information about locum tenens was gained by the author through research and a personal experience working as a locum tenens.

\section{Resources and Opportunities}

There are several options to consider when applying for a locum tenens position. These options include for-profit agencies, academic health centers, state agencies, federal sites such as the Indian Health Service, individual hospitals, and physicians or international staffing companies. There are advantages and disadvantages to each.

It is possible to locate open positions through advertisements in professional journals and on Web sites of various professional organizations, including the American Academy of Family Physicians and the American College of Physicians (Table 1). Internet search engines can be useful tools as well.

Although private arrangements can be made with a practice or physician, most placements for locum tenens occur through a national agency. These for-profit commercial companies are the largest locum tenens employers. There are 30 to 40 such companies in the United States, and it is estimated to be a billion dollar industry. ${ }^{1}$

Given the growth of the commercial industry, the National Association of Locum Tenens Organizations (NALTO) was formed in 2001 to help set standards for these for-profit companies as well as to serve as a resource for the health care industry. One important activity of this organization is to support an arbitration committee to handle conflicts between physicians, employers, and locum tenens companies. ${ }^{7}$

Domestic placements can last for as long as 1 day to several months. Medicare regulations, however, prohibit a locum tenens or other substitute physician from providing more than 60 continuous days of care in the United States. ${ }^{8}$

Although there is little information found in the literature about for-profit locum tenens companies, there has been some analysis of the programs offered by academic centers and state agencies. These organizations tend to be less costly for clinicians or practices seeking a locum tenens because the goal of these agencies is to provide a service to medically needy areas. Although they usually focus on the retention of established physicians and the recruiting of new ones, academic and state agencies also use locum tenens positions as a way to enhance and broaden the skills of residents and residency faculty. Residents and faculty who take locum positions can increase or vary their exposure to acute care, emergency medicine, obstetrics, and the realities of community practice. ${ }^{9-11}$

One of the most successful programs has been the University of New Mexico Locum Tenens Program. This program has been in existence since 1993 and is partially funded by the state legislature. In 2001 it provided services equivalent to 10 fulltime physicians and served 31 of 33 counties in the state. Forty residency graduates who worked in the program have gone on to accept positions in areas where they served. ${ }^{12}$ Other states, such as North Carolina and West Virginia, however, have not been as successful, and many promising programs no longer exist because of organizational difficulties and unstable funding.

Federal sites, such as the Indian Health Service, have long used locum tenens to fill vacant positions. An advantage to working at a federal site is that a state license for that particular site is not required. Instead, a physician can hold a valid license in any state and be able to practice as a locum tenens at any Indian Health Service site, which can save the physician both time and cost. For example, the physician can easily move from a reservation in New Mexico to one in Alaska.

The only international staffing company in the United States is Global Medical Staffing. Based in Utah, the company places locum tenens in underserved areas in Australia, New Zealand, the South Pacific, and the Caribbean. International placements differ from domestic placements in that 4 years of postgraduate experience is typically required. Because of international restrictions, doctors of osteopathy and midlevel providers are not 
eligible. Compensation is usually less than with for-profit companies in the United States. Travel, housing and transportation, however, are provided, and the income can be tax-free.

A potential advantage or disadvantage when working internationally is that placements tend to be longer. International assignments usually last at least 6 months because of the additional expenses related to relocation and travel.

\section{Points to Consider Before Signing a Contract}

It is important during the decision-making process to consider why a hospital or facility is seeking a locum tenens. Obviously, it is important to be wary if it is clear no previous physician wanted to stay in the position. There are, however, many practical and legitimate reasons why locum tenens are used. Recruiting and retention in underserved areas are major reasons. Hiring locum tenens gives local physicians needed time off and introduces potential clinicians to a new area where they might decide to linger or settle. Other reasons include covering time taken for continuing medical education, maternity leaves, or unexpected absences caused by illness. A locum tenens might be used to maintain a patient base while a permanent physician is being recruited or to accommodate seasonal changes in practice volume.

When seeking a locum tenens opportunity, there are certain things to consider. Duration of assignments can vary widely, and different practice sites can be assigned that will require moving even at weekly intervals.

Health insurance is typically not provided, either domestically or abroad, because a locum tenens is regarded as an independent contractor rather than an employee. Short-term health insurance is often inexpensive in foreign countries, however. A related issue to consider is that as an independent contractor, the locum tenens is paid only when working. There is no sick pay, and when the assignment ends, the income ends, too. An accountant can help anticipate tax costs, because federal, state, and social security taxes are not withheld.

There are some specific questions to ask both the staffing agency and those who work at the practice site (Table 2). ${ }^{13,14}$ Among the most important questions are why there is a vacancy and who is the person relating information. The level of detail and accuracy of information about the position
Table 2. Questions to Ask When Considering a Locum Tenens Position.

1. Review of contract: is there an "out" clause should the position be unsatisfactory?

2. What type of malpractice insurance, occurrence or claims made, is offered? What is the rating of the malpractice carrier?

3. What is the range of opportunities? This answer is particularly important if you want full-time work, yet the positions might be short-term. Are there positions for family physicians?

4. What is the credentialing process like? Does the agency handle licensing, hospital privileges, and so on? Is there a cost to the locum tenens?

5. Are 24-hour administrative support services by the company and risk management services available?

6. What is the payroll history of the company? How often does the locum tenens get paid and when will the physician receive the first paycheck? Will the agency pay even if the client does not pay them? Will the locum tenens be compensated for travel time, call, obstetrics, etc?

7. What are the specific responsibilities of the position? Ask about hours to be worked, call, obstetrics.

8. What are the available resources in the area? Are consultants readily available? What services does the hospital offer?

9. Especially in rural areas, how are patients transferred to higher level facilities? Will the locum tenens be expected to travel with patients on ground or air transports?

10. What happens in case of personal illness?

11. What type of accommodation will be provided?

Adapted from CompHealth ${ }^{13}$ and National Association of Physician Recruiters. ${ }^{14}$

might depend on whether the information is related by a recruiter, site administrator, or another physician who has already worked for the locum tenens company at that or a different site.

When obtaining information about the locum tenens position from another physician, it is important to inquire about that physician's background, level of training, and previous locum tenens experience. For example, the physician might be able to give advice on the scope and intensity of the work, such as intubating patients or performing other emergency procedures in a rural setting. A physician trained in a suburban or urban setting might not feel sufficiently comfortable with certain procedural skills to locate in a rural practice site.

\section{Conclusion}

Working as a locum tenens can be an exciting and rewarding opportunity. It is possible to meet interesting patients, learn about local culture, see how practices are organized, learn adaptability and 
broaden clinical skills. Even though the experience might be temporary, it is important to exercise some caution when choosing where to work by carefully checking workload and available resources.

A great deal is still unknown about the scope, demographics, and quality of care within the locum tenens industry. Unanswered questions about the effective use of locum tenens as a means of recruiting and retention in underserved areas remain as well and are areas of potential future research.

Peter Curtis, MD, guided the development of this article.

\section{References}

1. Locum tenens. Relief for rural health practitioners. Rural Health News 1996;Summer/Fall:5.

2. Krohnhaus AK. An organized locum tenens service and the cost of free time for rural physicians. Med Care 1981;19:1239-46.

3. Locum tenens. A staffing solution when physician supply does not meet demand. Available at: www. nejm.org/careerlinks. Accessed September 26, 2001.

4. Thompson E. Use of locum tenens grows. Modern Healthcare 2001;March:84.

5. Greene J. Growing number of locum tenens doctors strive for simpler life. Available at: www.amednews. com. Accessed September 26, 2001.
6. Leonhirth J. Road warriors. Available at: www. modernphysician.com/archive/article. Accessed September 26, 2001.

7. Grabner DL. Industry leaders join forces to raise the bar for physician staffing companies (press release). Salt Lake City, Utah: CompHealth, October 2, 2001.

8. Moore K. How to get reimbursed when a colleague treats your patients. Family Practice Manage 2000; $7: 14$.

9. Larsen LC, Derksen DJ, Garland JL, et al. Academic models for practice relief, recruitment, and retention at the University of New Mexico Medical Center and East Carolina University School of Medicine. Acad Med 1999;74(1 Suppl):S136-40.

10. Bergman J, Schneeweiss R. The locum tenens experience: a comparison with a university faculty practice. Fam Med 1985;17:103-5.

11. Rodney WM, Crown LA, Hahn R, Martin J. Enhancing the family medicine curriculum in deliveries and emergency medicine as a way of developing a rural teaching site. Fam Med 1998;30:712-9.

12. Derksen DJ, Powell W, Heider D, Clithero A, Akmajian P. The UNM Locum Tenens Program summary 1993 to 2001. Albuquerque, NM: UNM Center for Community Partnerships.

13. Beyond residency. Salt Lake City, Utah: CompHealth, 2001.

14. National Association of Physician Recruiters. What is locum tenens? Available at: www.napr.org. Accessed September 26, 2001. 